\title{
Serum creatine kinase isoenzymes in children with osteogenesis imperfecta
}

\author{
P. D’Eufemia ${ }^{1} \&$ R. Finocchiaro ${ }^{1} \&$ A. Zambrano ${ }^{1} \&$ V. $^{2}$ Lodato $^{1} \&$ L. Celli ${ }^{1} \&$ S. Finocchiaro ${ }^{1} \&$ \\ P. Persiani ${ }^{2} \&$ A. Turchetti ${ }^{1} \&$ M. Celli ${ }^{1}$
}

\section{Abstract}

Summary This study evaluates serum creatine kinase isoen- zyme activity in children with osteogenesis imperfecta to de- termine its usefulness as a biochemical marker during treatment with bisphosphonate. The changes of creatine kinase (CK) isoenzyme activity during and after discontinuation ther- apy were observed. These results could be useful in address- ing over-treatment risk prevention.

Introduction The brain isoenzyme of creatine kinase $(\mathrm{CKbb})$ is highly expressed in mature osteoclasts during osteoclasto- genesis, thus plays an important role in bone resorption. We previously identified high serum CKbb levels in 18 children with osteogenesis imperfect (OI) type 1 treated for 1 year with bisphosphonate (neridronate). In the present study, serum CK isoenzymes were evaluated in the same children with contin- uous versus discontinued neridronate treatment over a further 2-year follow-up period.

Methods This study included 18 children with OI type 1,12 with continued (group A) and 6 with ceased (group B) neridronate treatment. Auxological data, serum biochemical markers of bone metabolism, bone mineral density z-score, and serum total CK and isoenzyme activities were determined in both groups.

Results Serum CKbb was progressively and significantly in- creased in group A ( $<<0.004)$ but rapidly decreased to unde- tectable levels in group B. In both groups, the cardiac muscle

creatine kinase isoenzyme $(\mathrm{CKmb})$ showed a marked de- crease, while serum C-terminal telopeptide (CTx) levels were almost unchanged.Conclusions This study provides evidence of the cumulative effect of neridronate administration in increasing serum CKbb levels and the reversible effect after its discontinuation. This approach could be employed for verifying the usefulness of serum $\mathrm{CKbb}$ as a biochemical marker in patients receiving prolonged bisphosphonate treatment. Moreover, the decreased serum CKmb levels suggest a systemic effect of these drugs.

Keywords Bisphosphonates · Bone metabolism - Creatine kinase isoenzymes · Osteoclast · Osteogenesis imperfecta

Introduction 
Creatine kinase $(\mathrm{CK})$ is a dimeric enzyme that catalyzes the reversible reaction of creatine and adenosine triphosphate (ATP) to form phosphocreatine and adenosine diphosphate (ADP), a crucial reaction for cellular energy generation and metabolism [1]. The three known cytosolic CK isoenzymes include brain (CKbb), sarcomeric muscle (CKmm), and car- diac muscle $(\mathrm{CKmb})$ types [2]. CKbb is present in a range of tissues including the brain, retina, uterus, testes, and bone, in which it executes the function of energy maintenance and regulation [1].

It has been reported that the CKbb gene is highly expressed in rabbit osteoclasts [3] and that receptor activator of nuclear factor kappa-B ligand (RANKL) up-regulates CKbb during osteoclastogenesis [4]. Moreover, the decrease of CKbb ex- pression by RNA interference suppresses bone resorption by osteoclasts grown in vitro, showing that CKbb has a crucial role in bone remodeling [5].

In humans, elevation of serum levels of CKbb has been found in some types of osteopetrosis (OPT), a genetic disease in which osteoclasts fail to resorb bone; therefore, $\mathrm{CKbb}$ is proposed as a biochemical marker to distinguish true OPT from other sclerosing bone disorders [6-10]. Interestingly, se- rum CKbb has been reported to increase in patients affected by OPT as a consequence of bisphosphonate (BP) therapy $[11,12]$.

Osteogenesis imperfecta (OI) is a heritable, heterogeneous connective tissue disorder, causing bone fragility and several other connective tissue abnormalities with severities ranging from asymptomatic individuals to perinatal death. Osteoblasts in OI patients are unresponsive to mechanical load; therefore, they produce an abnormal bone matrix. To compensate for this, the osteoblast population increases alongside increased osteoclast activity, leading to a high bone turnover rate [13]. Other than bone fragility, OI is characterized by low bone mineral density (BMD), increased fracture rate, short stature, skeletal deformities, blue sclerae, dentinogenesis imperfecta, joint laxity, and deafness later in life [14]. On the basis of clinical, radiological, and genetic features, several OI types have been described [15], with type 1 being the mildest [16].

BPs are considered the treatment of choice in children with OI $[17,18]$. These synthetic analogs of inorganic pyrophosphate strongly inhibit bone resorption by sup- pressing the activity of osteoclasts and shortening their life span [19].

In 2004, we reported an increased serum CKbb level in 18 children with OI type 1 during the first year of treatment with neridronate [20]. Recently, BP treatment was reported to result in increased CK release from rabbit osteoclasts in vitro [21]. This result explains a possible mechanism for increased serum CK in patients treated with BPs [11, 20].

In the present study, we evaluated serum CK isoenzymes in the same 18 children used in our previous study during a further 2-year follow-up period [20]. The study included two groups: subjects who continued with neridronate treatment and subjects who discontinued neridronate treatment. The aim of this study therefore was to evaluate the effect of BP treatment on serum CK isoenzyme activity to determine its usefulness as a biochemical marker during the treatment of OI patients. 


\section{Subjects and methods}

Written informed consent was obtained from all patient par- ents prior to patient inclusion in the study. The study protocol was approved by the ethics committee of the Department of Pediatrics, Sapienza University of Rome.

This study included 18 children affected by OI type 1 who received 1 year neridronate treatment as described in our previous report [20]. Twelve children, five boys, and

seven girls (mean age [years] \pm standard deviation [SD]: $6.7 \pm 1.5$ ) continued neridronate treatment throughout the 2-year follow-up period (group A). Six children, four boys, and two girls (mean age [years] \pm SD: 5.2 \pm 3 ) discontinued neridronate treatment (group B) after reaching lumbar spine BMD (z-score) values of \pm 0.1 , in accordance with our protocol treatment of OI. All children were followed up for 2 years as outpatients in the Department of Pediatrics, Sapienza University of Rome. Exclusion criteria were the occurrence of fractures during the study, assumption of drugs, and/or acute illness inter- fering in bone metabolism. Neridronate (sodium neridronate, ABIOGEN PHARMA SpA, Pisa, Italy) was administered as a single infusion once every 3 months. Each infusion consisted of $2-\mathrm{mg} / \mathrm{kg}$ body weight, diluted in $100-\mathrm{mL}$ isotonic saline.

Baseline clinical and biochemical data of group A and group B are reported in Tables 1 and 2, respectively. Auxological data and serum biochemical markers of bone metabolism, including calcium, phosphate, intact parathy- roid hormone (iPTH), $25(\mathrm{OH})$ vitamin $\mathrm{D}(25(\mathrm{OH}) \mathrm{D})$, basal alkaline phosphatase (ALP), C-terminal telopeptide (CTx), lumbar spine radiographs, and BMD z-score, were determined in both groups at baseline and at 1 year in the previous study [20] and at 2 and 3 years during the follow-up period of the current study. Total serum CK and isoenzymes were analyzed in the same samples.

Serum biochemical studies

Total serum CK activity was measured in fasting blood sam- ples using the kinetic UV method (Instrumentation Laboratory SpA, Milano, Italy) optimized according to the Federation of Clinical Chemistry [22]. CK isoenzymes ( $\mathrm{mm}, \mathrm{mb}$, and $\mathrm{bb}$ ) were separated by electrophoresis on agarose gels. After elec- trophoresis, the sample strips were incubated for $1 \mathrm{~h}$ at $37^{\circ} \mathrm{C}$ with the reaction mixture. Catalytic activity was determined by spectrophotometric measurements as described previously [23].

Isoenzyme activity was expressed as international units per liter (U/L) (Helena Biosciences Europe, Sunderland, UK). Serum calcium, phosphate, ALP, iPTH, CTx, and 25(OH)D levels were measured using chemiluminescence and an auto- matic analyzer (Roche diagnostic SpS, Monza, Italy).

Radiological studies

BMD z-score was measured in the lumbar spine (L1-L4) by dual-energy x-ray absorptiometry 
using a Hologic QDR $4500^{\circ}$ system with reference values provided by the manufac- turer (Hologic, Bedford, MA) [24].

Statistics

Statistical differences in demographic, densitometric, and bio- chemical parameters were determined within groups. Comparisons between two time points in the same groups were carried out using the paired $t$ test. Because the data were approximately log-normally distributed, they were log- transformed before statistical analysis. Comparisons between more than two time points were performed using analysis of variance (ANOVA) for repeated measures. Results were expressed as means \pm SD. Significant deviations from the null hypothesis were stated when $\mathrm{p}<0.05$.

Results

Total serum CK and isoenzyme levels are reported in Tables 3 and 4 for continued (group A) and discontinued (group B) neridronate treatment, respectively. The data include the first year of treatment (0 to 1 year) published in our previous study [20] and 2-year observation time of the present study ( 1 to

3 years). Baseline data were determined before the first cycle of treatment and represent the pre-treatment values.

In group $\mathrm{A}$, serum $\mathrm{CKbb}$ showed a progressive increase during the 2-year follow-up that became statistically signifi- cant at the end of follow-up (3 vs 1 year; $p<0.004)$ with a mean increase rate of $4.4 \mathrm{U} / \mathrm{L} / \mathrm{yr}$. This change was less evident compared with the rapid increase observed in the previous study during the first year of neridronate treatment $(8.33 \mathrm{U} / \mathrm{L} / \mathrm{yr})$ (Fig. $1)$.

In Group B, serum CKbb exhibited a mild increase in all patients in the first year after discontinuing neridronate treatment, followed by a rapid decrease to undetectable levels at the end of the 2-year follow-up ( 3 vs 1 year; $p<0.001$ ). Furthermore, the values were lower than those observed at baseline ( 3 vs 0 year; $\mathrm{p}<0.001)$.

Regarding serum CKmb, group A showed a slight in- crease after 1 year of observation time followed by a marked decrease ( 3 vs 2 years; $p<0.005)$ and values were significantly lower compared with baseline (3 vs 0 year; p < 0.002) (Table 3, Fig. 1). In group B, serum CKmb tended to decrease from 1 year after discontinua- tion of neridronate treatment and was reduced to

undetectable level after the second year (3 vs 1 year; $p<0.002$ ) (Table 4, Fig. 1). In both groups, no significant changes were observed in the levels of CKmm isoenzyme (Tables 3 and $4)$.

Total serum CK showed a significant increase after 3 years of treatment compared with pretreatment but only in group A ( 3 vs 0 year; $p<0.05$ ). In both groups, no significant changes in 
total serum CK level were observed during follow-up in the present study.

Demographic, biochemical, and densitometric characteris- tics of group A and group B are reported in Tables 1 and 2, respectively. In group A, BMD z-score increased progressive- ly, while in group B there was a slight increase 1 year after discontinuing neridronate treatment, with a decrease at the end of follow-up.

CTx values in both groups remained almost unchanged during the 2-year follow-up time in the present study, with lower values in comparison with pre-treatment, which were comparable with normal range (Tables 1 and 2; Fig. 2). In both groups, lumbar spine radiographs showed no sign of osteosclerosis at the end of the evaluation period.

\section{Discussion}

In our previous study, we identified a significant increase in serum CKbb after 1 year of neridronate treatment in children affected by OI [20]. Because osteoclasts are considered a major target of the pharmacologic action of BPs, and are rich of cytosolic soluble CKbb, we postulated that this finding could reflect an osteoclast dysfunction inducing CKbb release. Recently, an in vitro study using BP-stimulated rabbit osteo- clasts supported that osteoclasts are the main source of $\mathrm{CK}$ release from the bone, and that this phenomenon is an osteo- clast apoptosis-related event [21]. These observations support the hypothesis that BP-induced osteoclast damage could ac- count for serum Ckbb increase in our OI-treated patients, sustaining the role of serum Ckbb as a marker of osteoclast dys- function [6-12].

In the present study, a significant serum CKbb increase was observed in the 12 children receiving continuous neridronate therapy after the 2-year follow-up (group A; 3 vs 0 year). However, the rate of this increase $(1.5 \mathrm{U} / \mathrm{L} / \mathrm{year})$ was lower than that observed during the first year of treatment ( $8.33 \mathrm{U} / \mathrm{L} /$ year). In vitro, $\mathrm{CK}$ release from osteoclasts exposed to BPs has been shown to be dose-dependent [21]. We therefore ex- pected a linear increase in serum CKbb over the study time due to the pharmacological effect of neridronate accumulation in the bone matrix. However, the aforementioned in vitro study also demonstrated that reduction of the osteoclast pop- ulation occurs when CK release is increased 2.6 times the basal value. Therefore, the reduced rate of serum CKbb in- crease observed in the present study could reflect reduction of the osteoclast population.

In group B, the level of serum Ckbb showed a mild in- crease after the first year of discontinued neridronate treat- ment, presumably reflecting the action of neridronate accumulation in the bone matrix during treatment. The rapid decrease to undetectable levels observed at the end of follow-up was surprising. In fact, compared with controls, untreated OI pa- tients showed slightly elevated serum CKbb levels, likely re- lated to increased bone turnover [20]. Therefore, the undetect- able CKbb levels observed 2 years after treatment discontin- uation seem to reflect the full suppression of osteoclast

apoptosis below pre-treatment level. This finding could repre- sent a mechanism finalized to a rapid recovery of the osteo- clast population. 
Regarding serum CTx, which reflects osteolytic activity by osteoclasts, we observed persistently lower levels in both groups in comparison with pre-treatment. To further evaluate these results, we compared them to the serum CKbb levels. In group A, the CTx values indicated that residual resorption activity was maintained after prolonged neridronate osteoclast exposure, even when a high apoptotic rate occurred, as indi- cated by elevated serum $\mathrm{CKbb}$ levels. Conversely, in group B, the CTx levels showed that neridronate continued to inhibit bone resorption 2 years after discontinuing treatment. This finding is in contrast with the undetectable serum CKbb levels observed at this time that reflect the recovery of the antiresorptive activity of osteoclasts. However, the aforemen- tioned in vitro study shows that $\mathrm{CK}$ release begins to increase following BP exposure resulting in $60 \%$ inhibition of CTx release [21]. Therefore, low-level BP exposure may occur, resulting in anti-resorptive activity not mediated by osteoclast

apoptosis. A similar situation could occur in vivo after discon- tinuation of treatment as a consequence of the continuous release of BPs accumulated in the bone matrix. This hypothesis is reasonable considering the two independent signaling pathways by which BPs exert their anti-resorptive and pro- apoptotic effects on osteoclasts [25, 26].

Taken together, these data show that neridronate exerts a high and constant inhibitory effect on osteoclast activity over a wide exposure-time range. Maximum anti-resorptive activity is obtained after the first year of treatment when CKbb release is augmented threefold. Furthermore, basal resorption activity is not abolished after long-term exposure when serum $\mathrm{CKbb}$ is strongly augmented. Moreover, neridronate continues to exert its maximal inhibition on bone resorption up to 2 years after discontinuation of treatment when serum $\mathrm{CKbb}$ is undetect- able. It is likely that serum CKbb has a different indication in relation to therapy. For example, in patients who have under- gone 1 year of treatment, a threefold increase seems to indicate the maximum inhibition of osteolytic activity, whereas in pa- tients who discontinued treatment, reduction of serum $\mathrm{CKbb}$

L/year) and lower increase in the further 2-year treatment (4.4 U/L/year) without evidence of plateau. Vertical bars represent SD. NER start start of neridronate treatment, NER stop stop of neridronate treatment. The shadow area shows the range of serum $\mathrm{CKbb}$ and $\mathrm{CKmb}$ values obtained from 20 healthy children matched for sex and age [20]

does not predict the recovery to pre-treatment levels of osteolytic activity. These concepts are only an inference of our results in the light of evidence emerging following in vitro studies. Further studies are needed to elucidate if they are useful for clinical purpose.

Another interesting finding from our study regards serum CKmb, the specific cytosolic cardiac isoform [2]. It is well known that BPs exert their specific action on bone resorption because they accumulate by affinity in the bone matrix and are solubilized prior to ingestion by osteoclasts during osteolytic activity $[25,26]$. In the previous report, we found that the pretreatment serum CKmb level was significantly higher com- pared with the control, although cardiovascular disease was excluded in the study population [20]. In the present study, we observed an intriguing reduction in serum $\mathrm{CKmb}$ in both groups during the second year of 
follow-up. These results suggest a possible systemic effect of BPs that implicates a different mechanism of entry through the plasma membrane in non-bone tissue cells. Moreover, it seems that neridronate, even after discontinuation, continues to exert an effect on

Osteoporos Int

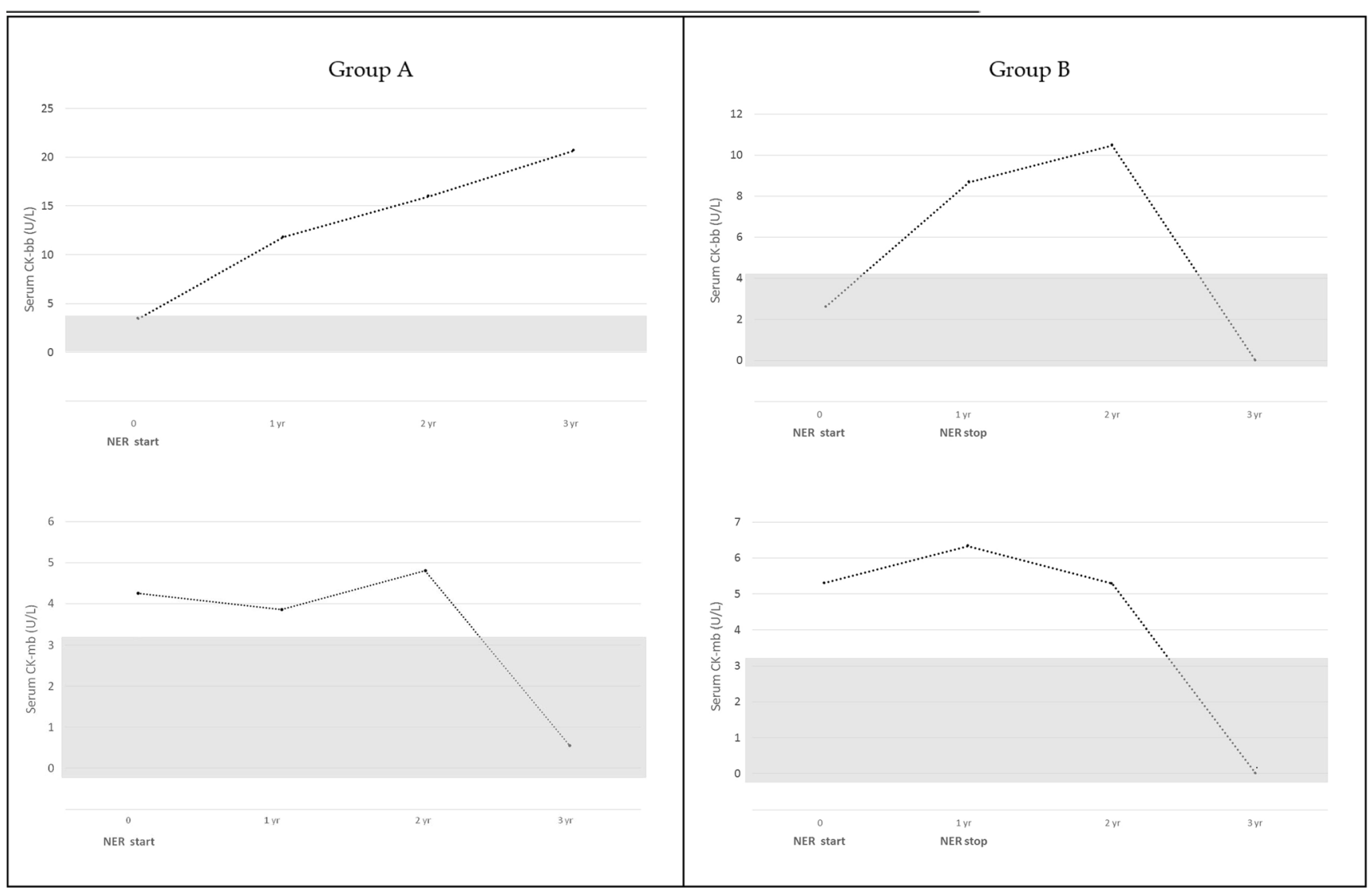

QS.PFEHEE-

Osteoporos Int 


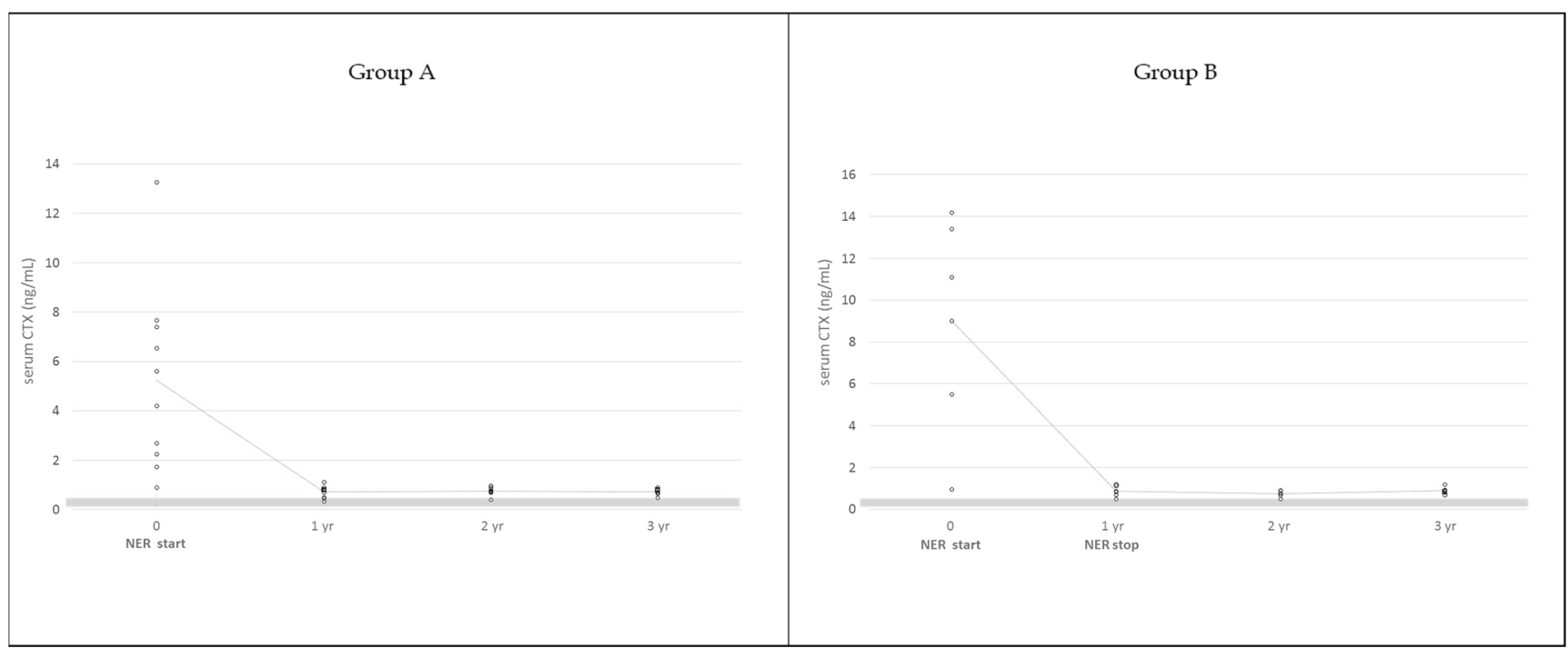

Fig. 2 Changes of serum CTX in 12 children who continued neridronate treatment (group A) and in 6 children who stopped neridronate treatment after 1 year (group B). The data include the first year of treatment (0 to 1 year) published in our previous study [20] and 2-year observation time

$\mathrm{CKmb}$ release by cardiomyocytes. Further studies are needed to elucidate if this observation is merely an epiphenomenon or could correlate with clinical cardiological parameters in these patients.

Our study has a number of limitations. First, the rela- tively small sample size limited the power of our statisti- cal analysis, restricting statistically significant correlations to be obtained with clinical parameters. Studies in larger cohorts (multicentric studies) are therefore required to bet- ter elucidate the usefulness of our results for clinical pur- pose. Second, we interpreted our in vivo results using evidence from an animal in vitro study that demonstrated the release of CK from cultured osteoclasts via the phar- macological action of BPs. Third, in the OI children who continued neridronate therapy, we observed a progressive increase of serum CKbb level without evidence of a pla- teau. In children who discontinued therapy, we did not observe recovery to pre-treatment levels at the end of the follow-up time. Therefore, further follow-up will be necessary to completely evaluate the variations of serum CKbb in relation to therapy.

In conclusion, this study provides evidence of the cu- mulative effect of neridronate administration in increasing serum CKbb levels and the reversible effect after its discontinuation. These data could be employed in verifying the usefulness of serum CKbb as a biochemical marker for clinical purpose in patients receiving prolonged BP treatment, especially regarding the prevention of over- treatment risk. This knowledge will be of particular inter- est considering the lack of consensus on criteria to initiate treatment, determine treatment duration, and evaluate

of the present study (1 to 3 years). Vertical bars represent SD. NER start start of neridronate treatment, NER stop stop of neridronate treatment. The shadow area shows the range of serum CTX normal values

long-term safety, especially in the pediatric population. Moreover, the decrease of serum 
CKmb suggests a sys- temic effect of these drugs, whose implications are not yet known, and needs to be investigated in future studies.

Compliance with ethical standards

Conflicts of interest

\section{References}

None.

1. Wallimann T, Wyss M, Brdiczka D, Nicolay K, Eppenberger HM (1992) Intracellular compartmentation, structure and function of creatine kinase isoenzymes in tissues with high and fluctuating energy demands: the 'phosphocreatine circuit' for cellular energy homeostasis. Biochem J 281:21-40

2. Wallimann T, Tokarska-Schlattner U (2011) The creatine ki- nase system and pleiotropic effects of creatine. Amino Acids 40:1271-1296

3. Sakai D, Tong HS, Minkin C (1995) Osteoclast molecular pheno- typing by random cDNA sequencing. Bone 17:111-119

4. Chen J, Sun Y, Mao X, Liu Q, Wu H, Chen Y (2010) RANKL up- regulates brain-type creatine kinase via poly (ADP-ribose) polymerase-1 during osteoclastogenesis. J Biol Chem 285:36315-36321

5. Chang EJ, Ha J, Oerlemans F, Lee YL, Lee SW, Ryu J, Hj K, et al. (2008) Brain-type creatine kinase has a crucial role in osteoclast- mediated bone resorption. Nat Med 14:966-972

6. Yoneyama T, Fowler HL, Pendleton JW, et al. (1989) Elevated levels of creatine kinase BB isoenzyme in three patients with adult osteopetrosis. N Engl J Med 320:1284-1285

QS.PFEAR -

Osteoporos Int

7. Gram J, Antonsen S, Hørder M, et al. (1991) Elevated serum level of creatine kinase BB in autosomal dominant osteopetrosis type II. Calcif Tissue Int 48:438-439

8. Yoneyama T, Fowler HL, Pendleton JW, et al. (1992) Elevated serum levels of creatine kinase bb in autosomal dominant osteopetrosis type II-a family study. Clin Genet 42:39-42

9. Whyte MP, Chines A, Silva DP Jr, et al. (1996) Creatine kinase brain isoenzyme (BB-CK) presence in serum distinguishes osteopetrosis among the sclerosing bone disorder. J Bone Miner Res 11:1438-1443

10. Waguespack SG, Hui SL, White KE, et al. (2002) Measurement of tartrate-resistant acid phosphatase and the brain isoenzyme of crea- tine kinase accurately diagnoses type II autosomal dominant osteopetrosis but does not identify gene carriers. J Clin Endocrinol Metab 87(5):2212-2217

11. Whyte MP, Wenkert D, Clements KL, et al. (2003) Bisphosphonate-induced osteopetrosis. N Engl J Med 349:457- 463

12. Whyte MP, McAlister WH, Novack DV, et al. (2008) Bisphosphonate-induced osteopetrosis: novel bone modelling de- fects, metaphyseal osteopenia, and osteosclerosis fractures after drug exposure ceases. $\mathbf{J}$ 
Bone Miner Res 23:1698-1707

13. Glorieux FH (2001) A disease of osteoblast. Lancet 358:S45

14. Sillence DO (1981) Osteogenesis imperfecta: an expanding pano- rama of variants. Clin Orthop 159:1125

15. Valadares ER, Carneiro TB, Santos PM, et al. (2014) What is new in genetics and osteogenesis imperfecta classification? J Pediatr 90: 536-541

16. Rauch F, Glorieux FH (2004) Osteogenesis imperfecta. Lancet 363: 1377-1385

17. Glorieux FH (2001) The use of bisphosphonates in children with osteogenesis imperfecta. J Pediatr Endocrinol Metab 2001 14(Suppl 6):1491-1495

18. Gatti D, Antoniazzi F, Prizzi R, et al. (2005) Intravenous neridronate in children with osteogenesis imperfecta: a randomized controlled study. J Bone Miner Res 20:758-763

19. Maruotti N, Corrado A, Neve A, Cantatore FP (2012) Bisphosphonates effects on osteoblast. Eur J Clin Pharmacol 68: 1013-1018

20. D'Eufemia P, Finocchiaro R, Villani C, Zambrano A, Lodato V, Palombaro M, Properzi E, Celli M (2014) Serum brain-type crea- tine kinase increases in children with osteogenesis imperfecta dur- ing neridronate treatment. Pediatr Res 75:626-630

21. Tanaka M, Mori H, Kayasuga R, Kawabata K (2015) Induction of creatine kinase release from cultured osteoclasts via the pharmaco- logical action aminobisphosphonates 3:4-59.

22. Schumann G, Bonora R, Ceriotti F, Clerc-Renaud P, Ferrero CA, Férard G, Franck PF, Gella FJ, et al. (2002) IFCC primary reference procedures for the measurements of catalytic activity concentration of enzymes at $37^{\circ} \mathrm{C}$. Part 2: references procedures for the measure- ments of catalytic concentration of creatine kinase. Clin Chem Lab Med 40:635-642

23. Trainer TD, Gruenig D (1968) A rapid method for the analysis of creatine phosphokinase isoenzymes. Clin Chim Acta 21:151-154

24. Zemel BS, Kalkwarf HJ, Gilsanz V, et al. (2011) Revised reference curves for bone mineral content and areal bone mineral density according to age and sex for black and non-black children: results of the bone mineral density in childhood study. J Clin Endocrinol Metab 96:3160-3169

25. Russell RG, Watts NB, Ebetino FH, et al. (2008) Mechanism of action of bisphosphonates: similarities and differences and their potential influence on clinical efficacy. Osteoporos Int 19:733-759

26. Matsumoto T, Nagase Y, Iwasawa M, Yasui T, Masuda H, Kadono Y, Nakamuratetsuro Y, et al. (2011) Distinguishing the proapoptotic and antiresorptive function of riseidronate in murine osteoclast. Arthritis \& Rheumatism 63:3908-3917 